\title{
Análise da estimulação cardíaca artificial atrioventricular universal (DDD) em comparação à ventricular (VVI): estudo multicêntrico
}

Roberto V. ARDITO*, Osvaldo T. GRECO*, Domingo M. BRAILE*, Paulo R. BROFMAN**, Ricardo ELOY***, Rubens MINILLOO ${ }^{* * *}$, Dirceu O. FAELLI Jr. ${ }^{* * * *}$, Aldo PESARINI ${ }^{* * * *}$, Rubens T. BARROS ${ }^{* * * * *}$, José Carlos S. ANDRADE******

ARDITO, R. V.; GRECO, O. T.; BRAILE, D. M.; BROFMAN, P. R.; ELOY, R.; MINILLO, R.; FAELLI Jr., D. O.; PESARINI, A.; BARROS, R. T.; ANDRADE, J. C. S. - Análise da estimulação cardiaca artificial atrioventricular universal (DDD) em comparaçâo à ventricular (VVI): estudo multicêntrico. Rev. Bras. Cir. Cardiovasc., 1(2):9-14, 1986.

RESUMO: Este trabalho analisa, em nosso meio, através das avaliaçóes radiológica, cicloergométrica, ecocardiográfica e também na eletrocardiografia dinâmica (Holter), o desempenho da estimulação DDD em relaçāo à VVI. Para isso, foi elaborado um estudo, do qual participaram 7 grandes Centros que implantam marcapasso no Brasil. No material estudado, năo se notou diferença significativa entre a estimulaçăo DDD e a estimulação VVI no paciente. Também não houve diferença quando se consideraram os pacientes chagásicos e não chagásicos.

DESCRITORES: estimulaçâo cardiaca, artificial; estimulaçâo cardiaca, técnicas: marcapassos cardiacos.

INTRODUÇÃO

A estimulação fisiológica, em que átrio e ventrículo são acoplados e ajustados em um intervalo $\mathrm{PR}$, consiste, atualmente, no mais avançado modo de estimulação cardiaca artificial.
Foi concebido há mais de 20 anos, porém, somente com o avanço tecnológico, tornou-se possível este modo de estimulação.

Este trabalho consiste em analisar, em nosso meio, através das avaliaçōes radiológica, cicloergo-

\footnotetext{
Trabalho realizado no Instituto de Moléstias Cardiovasculares. Sâo José do Rio Preto, SP, Brasil; no Hospital Evangélico de Curitiva, PR, Brasil; no Hospital Santa Izabel, Salvador, BA. Brasil; no Hospital Stella Maris de Guarulhos, SP, Brasil; na Santa Casa de Misericordia de Marilia, SP. Brasil e na Escola Paulista de Medicina, Sāo Paulo, SP, Brasil.

Apresentado ao 13: Congresso Nacional de Cirurgia Cardiaca, São Paulo, SP, 4 e 5 de abril, 1986

- Do Instituto de Moléstias Cardiovasculares de Sáo José do Rio Preto.

* Do Hospital Evangélico de Curitiba.

... Do Hospital Santa Izabel de Salvador.

.... Do Hospital Stella Maris de Guarulhos.

..... Da Santa Casa de Misericórdia de Curitiba.

...... Da Santa Casa de Misericórdia de Marilia.

........ Da Escola Paulista de Medicina.
}

Endereço para separatas: Roberto V. Ardito. Rua Castelo D'Água, 3030. Sâo josé do Rio Preto, SP, Brasil. 
ARDITO, R. V.; GRECO, O. T.; BRAILE, D. M: BROFMAN, P. R.; ELOY, R.; MINILLO, R.; FAELLI Jr., D. O.: PESARINI, A.; BARROS, R. T.; ANDRADE, J. C. S. - Análise da estimulação cardiaca artificial atrioventricular universal (DDD) em comparaçăo à ventricular (VVI): estudo multicêntrico. Rev. Bras, Cardiovasc., 1(2):9-14, 1986.

métrica, ecocardiográfica e, também, na eletrocardiografia dinâmica (Holter), o desempenho da estimulação fisiológica (DDD) em relação à ventricular de demanda (VVI).

Para tal, foi elaborado um protocolo comum, do qual participaram 7 grandes Centros que implantam marcapasso em nosso país.

\section{MATERIAL}

Dos pacientes operados; foram selecionados os 38 que tiveram o estudo completo da avaliação nos 6 diferentes Serviços, onde foram implantados marcapassos de dupla câmara (DDD) modelo Symbios (7005-7006), de maneira convencional.

Dos 38 pacientes, 23 são homens e 15 são mulheres, com idade de 12 a 77 anos de variação, com idade média de 54 anos (Gráfico 1).

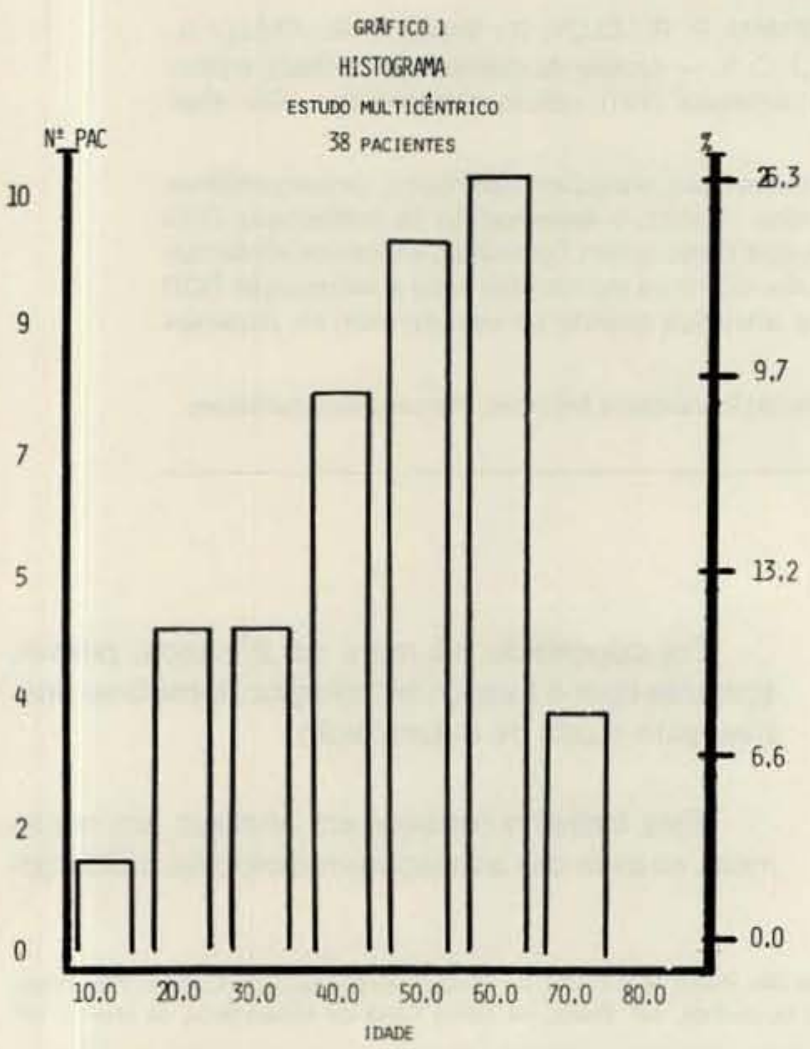

Os pacientes chagásicos consistiam $50 \%$, com média etária de 48 anos, e os não chagásicos, 52 anos (Quadro 1, Gráficos 2 e 3).

$\mathrm{O}$ índice cardiotorácico (ICT) ficou entre 0,4 a 0,57 , sendo $68,4 \%$ com ICT de 0,50 a 0,54 (Quadro 2).
As indicações foram de: doença do nó sinusal, 20 pacientes $(52 \%)$; BAV total 11 pacientes $(29 \%)$; BAV 2: tipo II, 6 pacientes (16\%) e síndrome braditaqui, 1 paciente (3\%) (Quadro 3).

\section{QUADRO 1}

ESTUDO MULTICÊNTRICO DDD X VVI

\begin{tabular}{c}
\hline TOTAL DOS PACIENTES $=38$ \\
\hline$\delta \cdot 23 \quad q \cdot 15$ \\
MÉDIA DE IDADE $=51$ ANOS \\
VARIAÇAO: $12 \cdot 77$ \\
CHAGÁSICOS $=19 \quad \mathrm{M}=48$ ANOS \\
N CHAGÁSICOS $=19 \quad \mathrm{M}=52$ ANOS \\
\hline
\end{tabular}

\section{QUADRO 2}

ÍNDICE CARDIO-TORÁCICO DISTRIBUIÇÃO DA FREQŨÊNCIA

\begin{tabular}{lccc}
\hline \hline INTERVALO & FREQŪENCIA & $\%$ & $\%$ ACUMULADA \\
\hline $0,400-0,449$ & 1 & 2,6 & 2,6 \\
$0,450-0,499$ & 5 & 13,2 & 15,8 \\
$0,500-0,549$ & 26 & 68,4 & 84,2 \\
$0,550-0,600$ & 6 & 15,8 & 100,0 \\
\hline
\end{tabular}

\section{GRAFICO 2}

HISTOGRANA

ESTUDO MULCITÊNTRICO

19 PACIENTES

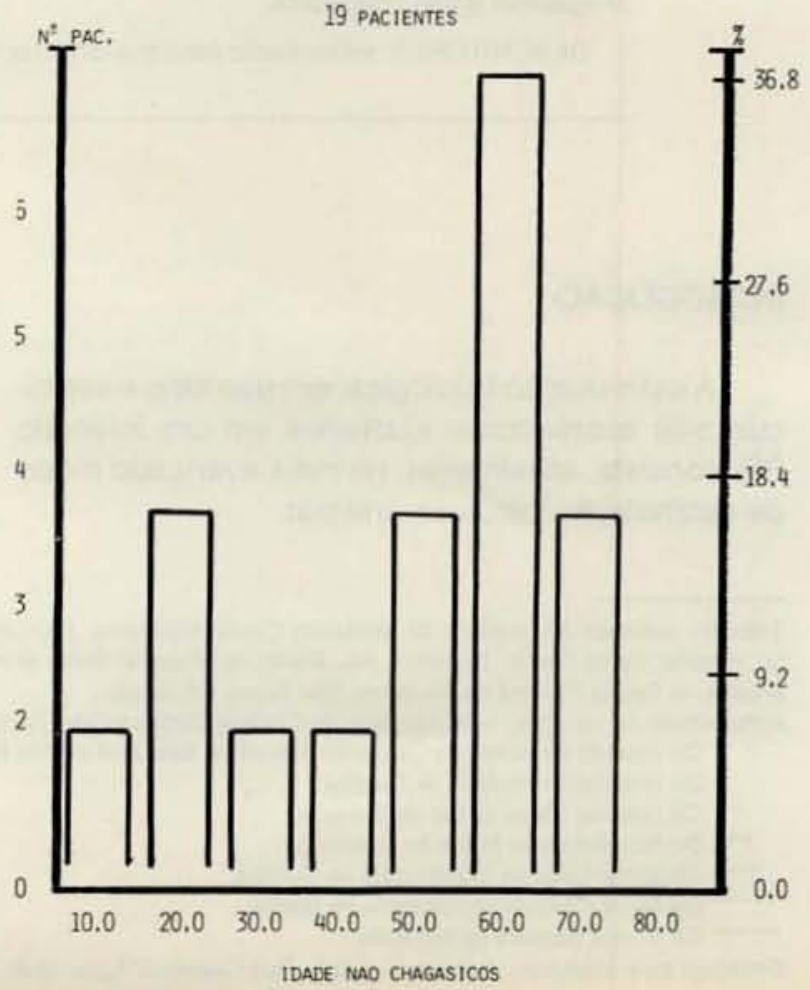


ARDITO. R. V.; GRECO, O. T.; BRAILE, D. M: BROFMAN, P. R.; ELOY, R.; MINILLO, R.; FAELLI Jr., D. O.: PESARINI, A.; BARROS, R. T.: ANDRADE, J. C. S. - Análise da estimulação cardiaca artificial atrioventricular universal (DDD) em comparaçāo à ventricular (VVI): estudo multicèntrico. Rev. Bras. Cardiovasc., 1(2) 9-14, 1986.

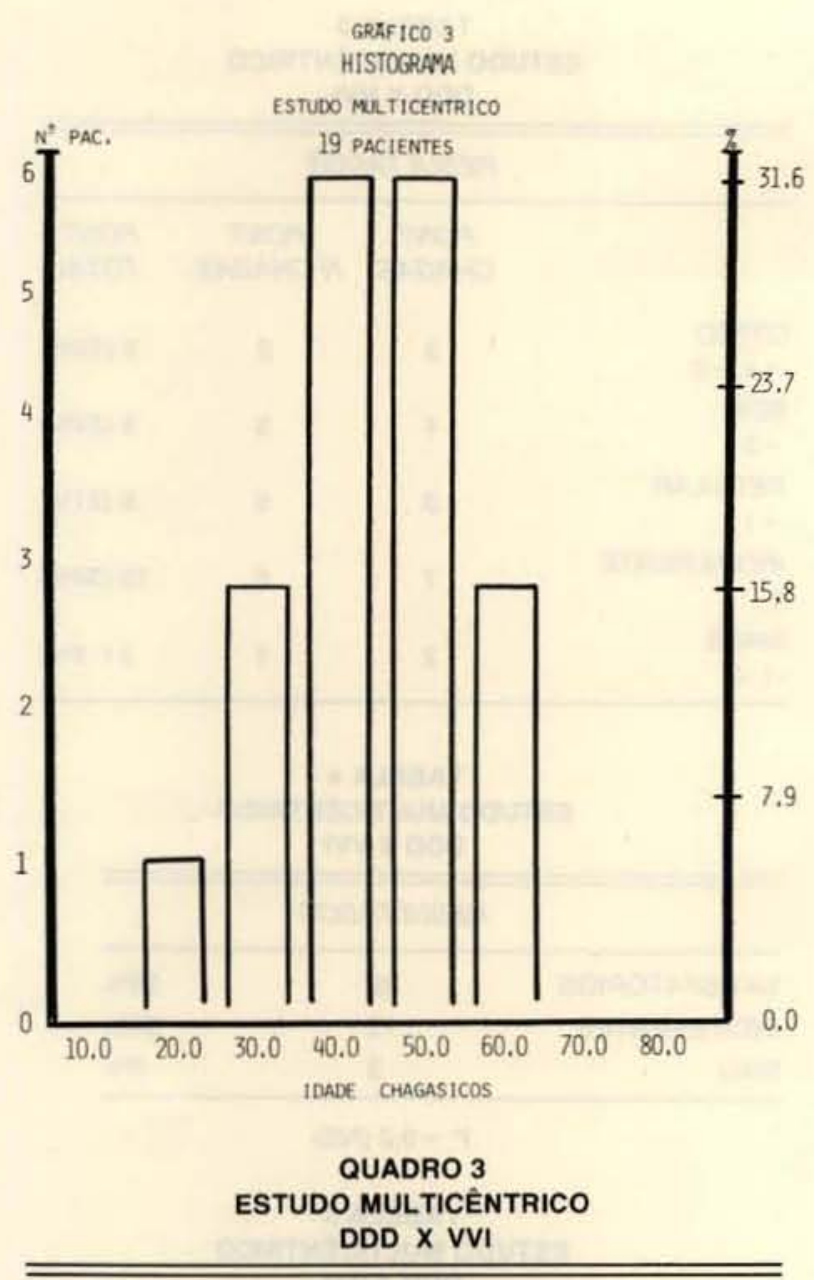

INDICACAOA 38 PACIENTES

CHAG. N CHAG. TOTAL

\begin{tabular}{lrrr} 
DOENÇA DO NO SINUSAL & 10 & 10 & $20(52 \%)$ \\
BAV 2: T. II & 2 & 4 & $6(16 \%)$ \\
BAVT & 7 & 4 & $11(29 \%)$ \\
SIND. BRADITAQUI & 0 & 1 & $1(3 \%)$ \\
\hline
\end{tabular}

\section{MÉTODO E ESTUDO}

Foi, primeiramente, elaborado um protocolo, para que os Serviços pudessem implantar e estudar os pacientes com este tipo de marcapasso e que todos tivessem os mesmos critérios no implante, na seleção dos pacientes e, também, na avaliação dos estudos clínicos, radiológicos, cicloergométricos, ecocardiográfico e na eletrocardiografia dinâmica, onde foi observado o desempenho na estimulação DDD em relaçāo à VVI.

Todos os pacientes foram colocados, em períodos intercalados de 2 meses, em DDD e VVI e realizados os exames de avaliação, segundo esquema abaixo*.

A avaliação clínica consistiu na observação de uma lista dos principais sinais e sintomas, bem como a medicação que o paciente recebia nos dois modos de estimulação, classificando-se em diferentes classes funcionais.

A cicloergometria consistiu na avaliação da quantidade de trabalho executado nos diferentes modos de estimulação. $\mathrm{Na}$ ecocardiografia bidimensional, deu-se a atenção à fração de ejeção do VE medida no módulo $\mathrm{M}$ em repouso.

$\mathrm{Na}$ eletrocardiografia dinâmica (Holter), foram observados a quantidade de extrassístoles ventriculares e o desempenho do MP em DDD e VVI.

$\mathrm{Na}$ avaliação geral, foram dadas notas de +1 , 0 e -1 no desempenho do marcapasso em DDD $x$ VVI. Por exemplo: se um determinado paciente tivesse um melhor desempenho do teste ergométrico em DDD em relação à VVI, ele receberia nota +1. Se não tivesse sido observada diferença signi-

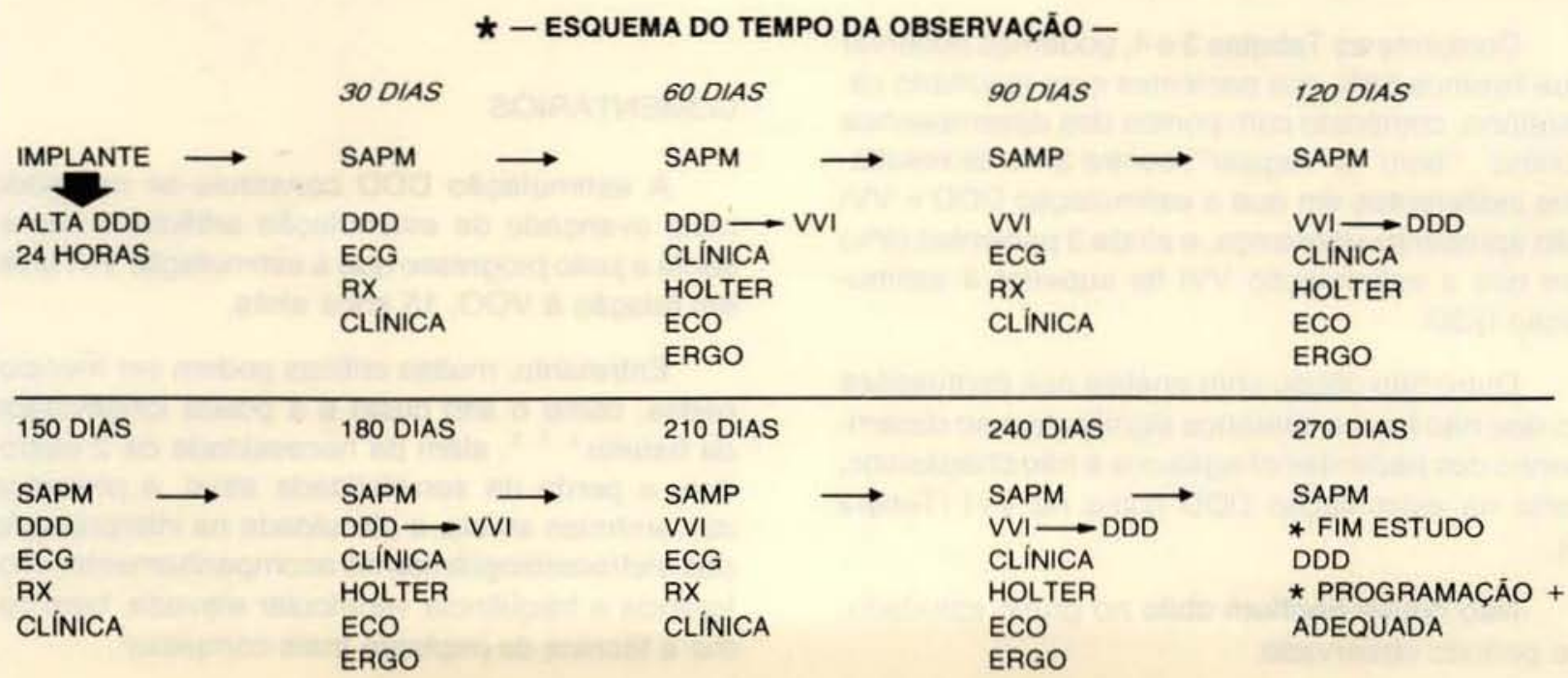


ARDITO, R. V.; GRECO, O. T.; BRAILE, D. M: BROFMAN, P. R.; ELOY, R.; MINILLO, R.; FAELLI Jr., D. O.; PESARINI, A.: BARROS, R. T.: ANDRADE. J. C. S. - Análise da estimulaçāo cardiaca artificial atrioventricular universal (DDD) em comparação à ventricular (VVI): estudo multicêntrico. Rev. Bras, Cardiovasc., 1(2):9-14; 1986.

ficativa, a nota seria 0 . E se o marcapasso em estimulação VVI fosse melhor que a estimulação DDD, executando maior quantidade e trabalho, receberia nota -1 .

Os resultados obtidos nos diferentes testes de avaliação da estimulação DDD em relação à VVI foram classificados de acordo com a pontuação obtida em: +4 e +3 (ótimo); + 2 (bom); +1 (regular); 0 (indiferente); -1 (mau). (Tabelas 1 e 2 ).

TABELA 1

ESTUDO MULTICÊNTRICO DDD X VVI

\begin{tabular}{lrrr}
\hline \hline PONTUACAO & +1 & 0 & -1 \\
\hline ERGO & 12 & 21 & 5 \\
ECO & 10 & 26 & 2 \\
HOLTER & 7 & 26 & 5 \\
CLINICA & 22 & 14 & 2 \\
\hline
\end{tabular}

TABELA 2

ESTUDO MULTICÊNTRICO DDD X VVI

\begin{tabular}{lrrrrrr}
\hline \hline $\begin{array}{c}\text { CHAGASICO } \\
\text { NAO CHAGASICO }\end{array}$ & & & & & & \\
PONTUACAO & +1 & 0 & -1 & +1 & 0 & -1 \\
\hline ERGO & 6 & 10 & 3 & 6 & 13 & 0 \\
ECO & 5 & 13 & 1 & 5 & 13 & 0 \\
HOLTER & 4 & 13 & 3 & 5 & 13 & 3 \\
CLINICA & 9 & 9 & 1 & 13 & 5 & 1 \\
\hline
\end{tabular}

\section{RESULTADOS}

Conforme as Tabelas 3 e 4, podemos observar que tivemos $58 \%$ dos pacientes com resultado satisfatório, composto com pontos dos desempenhos "ótimo", "bom" e "regular", contra $34 \%$ de resultados indiferentes em que a estimulação DDD e VVI nāo apresentou diferença, e ainda 3 pacientes $(8 \%)$ em que a estimulaçāo VVI foi superior à estimulação DDD.

Outro fato obtido com análise das pontuaçōes foi que não houve diferença significativa no desempenho dos pacientes chagásicos e não chagásicos, tanto na estimulaçāo DDD como na VVI (Tabela 5).

Não houve nenhum óbito no grupo estudado. no período observado.
TABELA 3

ESTUDO MULTICÊNTRICO DDD X VVI

\begin{tabular}{|c|c|c|c|}
\hline & \multicolumn{2}{|c|}{ RESULTADOS } & \multirow[b]{2}{*}{$\begin{array}{l}\text { PONT: } \\
\text { TOTAL }\end{array}$} \\
\hline & $\begin{array}{l}\text { PONT. } \\
\text { CHAGAS. }\end{array}$ & $\begin{array}{c}\text { PONT. } \\
\text { N CHAGAS. }\end{array}$ & \\
\hline $\begin{array}{l}\text { OTIMO } \\
+4+3\end{array}$ & 3 & 2 & $5(13 \%)$ \\
\hline $\begin{array}{l}\text { BOM } \\
+2\end{array}$ & 4 & 5 & $9(24 \%)$ \\
\hline $\begin{array}{l}\text { REGULAR } \\
+1\end{array}$ & 3 & 5 & $8(21 \%)$ \\
\hline $\begin{array}{l}\text { INDIFERENTE } \\
0\end{array}$ & 7 & 6 & $13(34 \%)$ \\
\hline $\begin{array}{l}\text { MAUS } \\
-1-2\end{array}$ & 2 & 1 & $3(8 \%)$ \\
\hline
\end{tabular}

TABELA 4

ESTUDO MULTICÊNTRICO DDD X VVI

\begin{tabular}{lcr}
\hline \hline & RESULTADOS \\
\hline SATISFATÓRIOS & 22 & $58 \%$ \\
INDIFERENTES & 13 & $34 \%$ \\
MAU & 3 & $8 \%$ \\
\hline
\end{tabular}

$P=0,2$ (NS)

TABELA 5

ESTUDO MULTICÊNTRICO

DDD X VVI

\begin{tabular}{lrr}
\hline \hline RESULTADOS: CHAGAS & 19 & NAOO CHAGAS $=19$ \\
\hline SATISFATORIO & 10 & 12 \\
INDIFERENTE & 7 & 6 \\
MAU & 2 & 1 \\
\hline
\end{tabular}

$P=0,5$ (NS)

\section{COMENTÁRIOS}

A estimulação DDD constituiu-se no modo mais avançado de estimulação artificial e representa o justo progresso que a estimulação VVI teve, em relação à $\mathrm{VOO}, 15$ anos atrás.

Entretanto, muitas críticas podem ser mencionadas, como o alto custo e a pouca longevidade da bateria ${ }^{2,7}, 8$, além da necessidade de 2 eletrodos, a perda da sensibilidade atrial, a presença das arritmias atriais, a dificuldade na interpretaçāo dos eletrocardiogramas no acompanhamento, intolerância a freqüência ventricular elevada, bem como a técnica de implante mais complexa ${ }^{2}$. 
ARDITO, R. V.: GRECO, O. T.; BRAILE, D. M; BROFMAN, P. R.; ELOY, R.; MINILLO, R.; FAELLI Jr., D. O.; PESARINI. A.: BARROS, R. T.; ANDRADE, J. C. S. - Análise da estimulaçảo cardiaca artificial atrioventricular universal (DDD) em comparaçấo à ventricular (VVI): estudo multicêntrico. Rev. Bras, CardiovasC, 1(2):9-14. 1986

Revendo a literatura, os autores tentam demonstrar que a estimulação DDD é superior à VVI: no entanto, esta demonstraçāo nāo tem sido convincente.

Em estudos ergométricos onde se comparou a capacidade funcional, além do consumo de oxigênio, não houve diferença significativa da estimulação DDD em relação à VVI, mas uma melhor adaptaçāo durante o exercício em $\mathrm{DDD}^{3}$.

Com relação a estudos de termodiluição para determinar o débito cardíaco, os pacientes com ICC grave melhoraram com a estimulação VVI, quando comparados com os de VE normal ${ }^{5}$.

As pesquisas de ALT et alii (Munique) ${ }^{1}$ comprovam que 0 aumento significante de tolerância ao exercicio depende mais do aumento da freqüência no exercício do que do sincronismo átrioventricular.

No material estudado, não houve diferença significativa entre a estimulaçāo DDD e a estimulação VVI no paciente. Também não houve diferença quando se consideraram os pacientes chagásicos e não chagásicos.

\section{CONCLUSĀO}

No material estudado, não foi constatada diferença significativa entre a estimulação $D D D$ e a estimulação VVI, nos pacientes; além disso, também não houve diferença, quando se consideraram os pacientes chagásicos e não chagásicos.

\section{RBCCV}

ARDITO, R. V.; GRECO, O. T.; BRAILE, D. M.; BROFMAN, P. R.; ELOY, R.; MINILLO, R.; FAELLI Jr. D. O.; PESARINI, A.; BARROS, R. T.; ANDRADE, J. C. S. - Comparative analysis of universal atrio-ventricular (DDD) vs ventricular (VVI) artificial cardiac stimultion: a multicenter study. Rev. Bras. Cir. Cardiovasc, 1 (2):9-14, 1986

ABSTRACT: This paper documents, through radiological, stress test, echocardiography and Holter monitor, the effect of DDD and VVI stimulation. Seven Centers with large experience in pacemakers contributed cases. We didn't notice significant differences between DDD and VVI stimulation in the patients studied and in Chagas or non-Chagas disease.

DESCRIPTORS: cardiac stimulation, artificial; cardiac stimulation, techniques; pacemakers, cardiac

\section{REFERÊNCIAS BIBLIOGRÁFICAS}

1 ALT, E.; KRIEG, J.; VÖLKER, R. - Long term results in 127 patients with physiological pacemakers (phPM). PACE, 8:A-35, 1985.

2 DODINOT, B - Dual chamber pacing modes in cardiac pacing. In: Proceedings of the XII World Symposium on Cardiac Pacing. Viena, maio 1983. p. 449.

3 GODIN, J.F.; POTIRON-JOSSE, M.; LE MAREC, H.; LOUVET, S.; L'HÉNAFF, H. W. - Oxygen uptake during stress testing ind DDD versus VVI pacing. PACE, 8:A-34, 1985.

4 GRECO, O. T.; ARDITO, R. V.; LORGA, A. M.; RIBEIRO, R. A.; BELLINI, A. J.; BILAQUI, A.; NICOLAU, J. C.; JACOB, J. L. B.; AYOUB, J. C. A.; ANGELONI, M. A.; LIMA, E. R.; ANSELMO, E. F.; FEDOZZI, N. M.; BRAILE, D. M. - O marcapasso cardiaco artificial deve ser multiprogramável?: estudo em 931 pacientes. Arq. Bras. Cardiol., 45 (Supl. 1): 105, 1985. (Resumo)
5 KOURKOULAKOS, C.; GIALAFOS, J.; PARASKEVAS, P.: TSAKIRIS, M.; KREMASTINOS, D.; TOUTOUZAS, P - Assessment of left ventricular (LV) function in ventricular and $A-V$ pacing using systolic time intervals and thermodilution technique. PACE, 8: A-34, 1985.

6 PINTO, G. H. C.; LORGA, A. M.; AYOUB, J. A. C.; CURY, M. V. A. R.; GRECO, O. T.; NICOLAU, J. C.; JACOB. J. L. B.; ARDITO, R. V.; GARZON, S. A. C.: BELLINI, A. J.; RIBEIRO, R. A.; BILAQUI, A.; BRAILE, D. M. - Marcapasso atrial transesofágico eficácia diagnóstica e terapêutica. Arq. Bras. Cardiol., 45 (Supl. 1) 107, 1985. (Resumo)

7 POTVIN, E. A.; GOLDMAN, B. S.; DUNCAN, J. A.; TONG. C. P.; SCHOICHET, M.; CAMERON, D. A. - Morbidity and mortality during follow-up of patients with dual chamber pacemakers. PACE. 8: A-36, 1985.

8 YAMAGATA, S.; MATSUMURA, K.; OHYAGI, K.; IKEDA K.: ISHIGURO, T.; MATSUMOTO, K.; MATSUSHITA T.; TAKENOSHITA, T. - An application of the dualchamber pacemaker to a single chamber pacing: a preliminary report of VVD Mode. PACE, 8: A-36, 1985. 
ARDITO, R. V.; GRECO, O. T.; BRAILE, D. M; BROFMAN, P. R.; ELOY, R.; MINILLO, R.; FAELLI Jr., D. O.; PESARINI, A.; BARROS, R. T.; ANDRADE, J. C. S. - Analise da estimulação cardiaca artificial atrioventricular universal (DDD) em comparaçāo à ventricular (VVI): estudo multicêntrico. Rev. Bras, Cardiovasc., 1(2):9-14, 1986.

\section{Discussão}

\section{DR. PAULO GAUCH}

SAO Paulo, SP

Desejo agradecer à Comissăo Organizadora do 13: Congresso Nacional de Cirurgia Cardiaca a indicação do meu nome como comentador oficial deste trabalho. Os resultados obtidos neste estudo multicêntrico não diferem do que pensamos em relação às vantagens ou benefícios de estimulação DDD em relação à VVI. Baseados na apresentação do Dr. Ardito, observamos que $37 \%$ dos pacientes se beneficiaram com a estimulação DDD, em comparação à VVI. No entanto, $63 \%$ não obtiveram benefícios significativos com o tipo de estimulação de dupla câmara. A nossa meta é individualizar os pacientes que realmente se beneficiam com a estimulação DDD, classificando-os conforme a etiologia, o tipo de distúrbio da condução que induziu ao implante do marcapasso, a presença, ou não, de insuficiência cardíaca, arritmias, etc. Inúmeros trabalhos, na literatura, demonstram que o implante de marcapasso VVI devolve qualidade e quantidade de vida à grande maioria de pacientes que recebem este tipo consagrado de estimulação. No entanto, o fato de um implante de marcapasso VVI devolver ao paciente a expectativa de vida de uma populaçăo normal, nāo afasta a possibilidade de permanência ou persistência de sintomas. Estes sintomas podem persistir pela própria patologia de base, ou serem originados pela perda do sincronismo átrioventricular e os fenômenos decorrentes (Slide). Observamos, neste diapositivo, que os pacientes com bloqueio da condução átrioventricular, mesmo após o implante de marcapasso VVI, possuem uma porcentagem maior de sintomas decorrentes da miocardiopatia associada, enquanto que - grupo de pacientes com disfunção sinusal apresentam uma porcentagem significativa de sintomas relacionados com a síndrome do marcapasso (Slide). Portanto, no grupo de pacientes com bloqueio plante de marcapasso DDD se justificaria para meda condução átrioventricular, a indicação de imIhorar a capacidade física em pacientes jovens; nos bloqueios $\mathrm{A}-\mathrm{V}$ associados à insuficiência coronária, obtendo-se a queda do consumo de oxigênio do miocárdio e nos bloqueios $\mathrm{A}-\mathrm{V}$ associados e anomalias congênitas e afecçōes valvares, procurando-se obter melhor condição hemodinâmica no território pulmonar (Slide). Já as indicaçōes nas disfunçōes sinusais são mais amplas, devendo-se, obrigatoriamente, indicar o DDD como modo de estimulaçăo em pacientes portadores de síndrome do marcapasso; na doença intrínseca do nó sinusal associada a distúrbios da condução $A-V$ e presença de condução retrógrada, e na forma cárdio-inibitória da hipersensibilidade do seio carotídeo pelo alto risco de bloqueios da condução $\mathrm{A}-\mathrm{V}$ e alta incidência de condução retrógrada associada (Slide). Concluímos nosso comentário salientando que a estimulação DDD pode trazer benefícios para grupos selecionados de pacientes, devendo-se, por outro lado, evitar-se o seu uso indiscriminado, visto que os benefícios da estimulação VVI satisfazem a maioria dos pacientes.

\section{DR. ARDITO}

(Encerrando)

Gostaria de agradecer ao Dr. Paulo Gauch, pelos comentários, e salientar que o marcapasso DDD deve ser colocado em pacientes que apresentem uma boa resposta atrial ao esforço, com implemento de freqüência, e ai teríamos a situação ideal, com o sincronismo átrioventricular. Outro fato importante que devemos frizar é que o paciente não chagásico tem as mesmas manifestações de resposta ao marcapasso que o paciente chagásico. Quero deixar, aqui, mais uma vez, aos meus colegas que colaboraram com este estudo multicêntrico, os agradecimentos em nome de todos e, ao Dr. Naim Savaia, pela orientação estatística. 